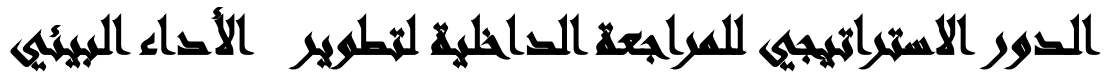

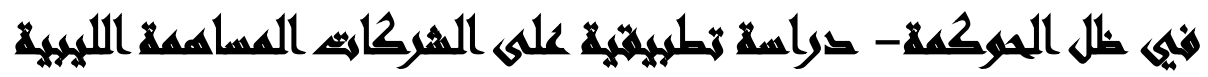 [r)]

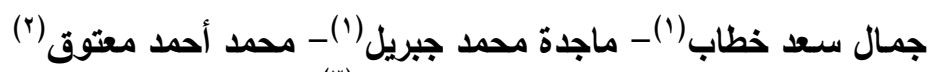

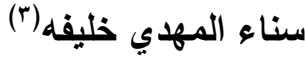

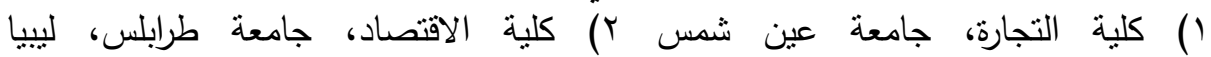 r) كلية الاقتصاد والعلوم السياسية، جامعة بني وليد، ليبيا. \\ المستخلئ
}

تهدف هذه الدراسة إلى نوضيح الدور الاستراتيجي للمراجعة الداخلية لنطوير الأداء البيئي

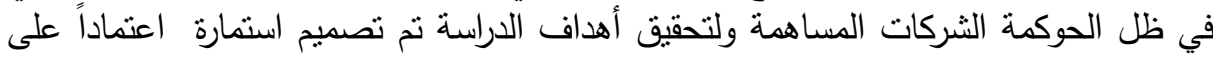

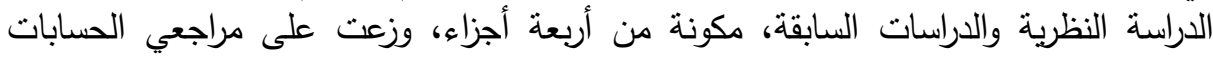

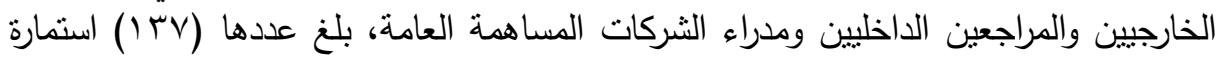

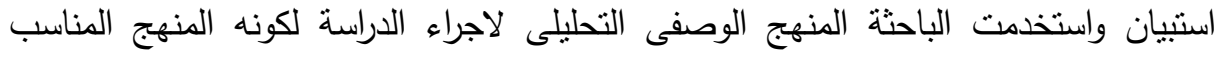
لدراسة الظواهر الاجتماعية واستخدم البرنامج الإحصائي للاراسات الاجتماعية (SPSS) لتحليل لإنيل

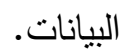

وقا خلصت الاراسة إلى نتائج عديدة نذكر منها:

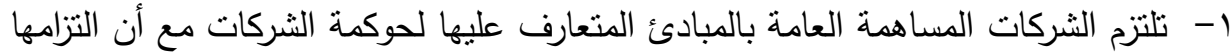

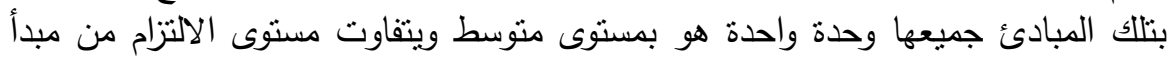

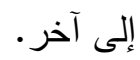

r- لا توجد في ليييا لجان مراجعة في الشركات المساهمة العامة باستثناء البنوك الخاضعة

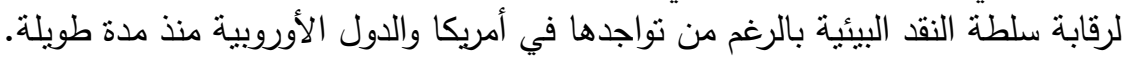

وقدمت الباحثون مجموعة من التوصيات أهمها:

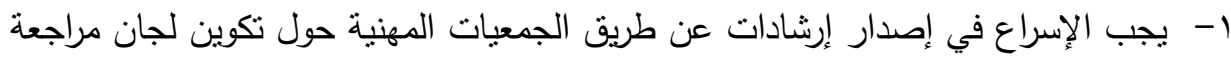

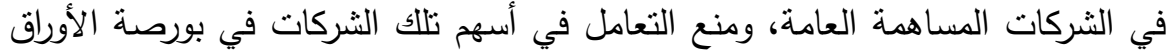
المالية إذا لم تقم بتكوين لجان المراجعة بها.

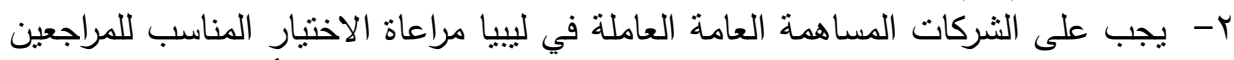

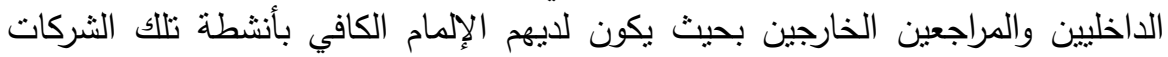

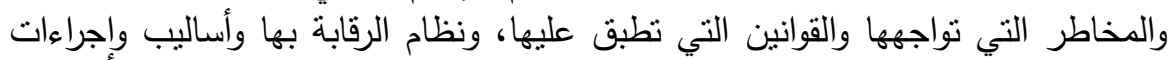

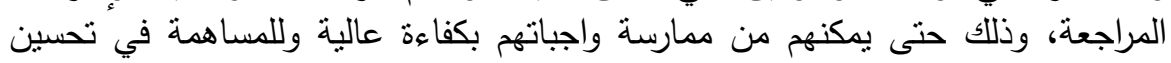

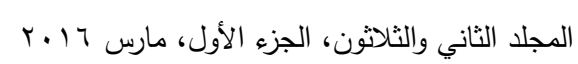




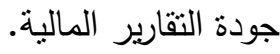

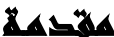

تزايد الاهتمام بموضوع حوكمة الثركات في السنوات الأخيرة خاصة بعد انهيار بعض الثركات العالمية الكبرى في مناطق مختلفة من أبرزها: (أنرون، وورلد كوم) في الولايات المتحدة الأمريكية، وما ترتب على ذلك من حدوث أزمة مالية ثقة عالمية في القوائم المالية للشركات بسبب ضعف الثفافية والإفصاح المحاسبي مع انتشار استخدام أسلوب المحاسبة

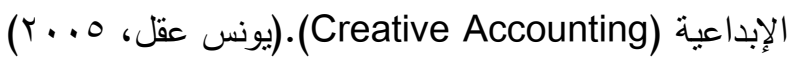
وقد سارعت كثير من الدول والمنظمات العالمية التي تنتبى مفهوم حوكمة الثركات وتعزيز الإفصاح والثفافية ومنع وقوع مزيد من الأزمات المالية مستقبلاً، وانطلاقاً من هذه منها

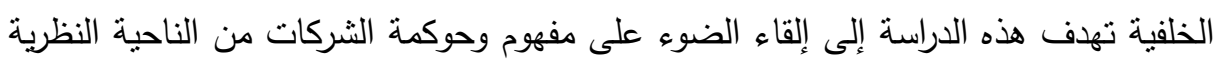
والتطبيقية، والتعرف على أسباب ودوافع انتتار هذا المفهوم في السنوات الأخيرة في الدول المتقدمة، والتي جاءت لتمنل الحل الأمنل لمعالجة أسباب الانهيار وأزمة الثقة التي ترتب عليه والتي طالب تأثيرها أغلب التعاملات في الأسواق العالمية والتي كانت من أهم مسبباتها

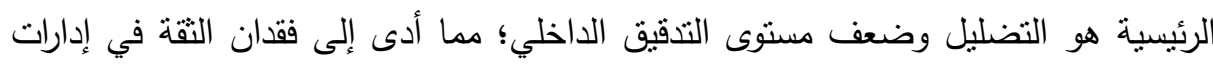

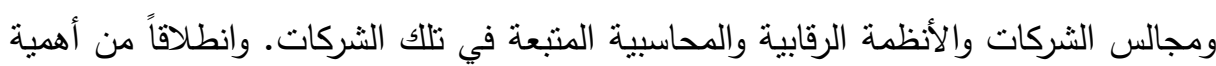
حوكمة الثركات وضرورة نقلها حيز التطبيق فإن التدقيق الداخلي يُعد أحد ركائز هذا التطبيق فئنس

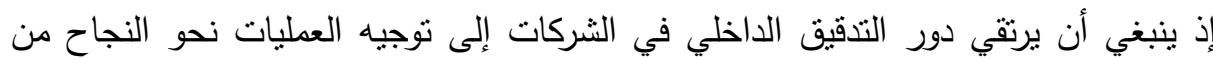

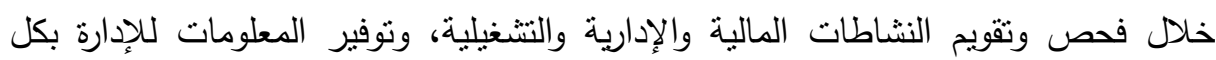
مستوياتها لمساعدتها في تتفيذ هذه الاستراتيجيات بشكل صحيح، وكذلك تفعيل دور التدقيق ونتئ

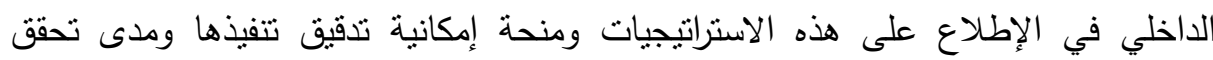

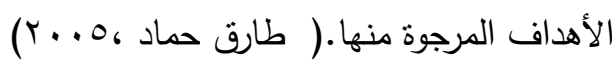

وقد أظهرت السنوات السابقة اقتصاديات العديد من الدول قد تعثرت نتيجة افتقارها إلى الحاكمية الجيدة؛ مما ألحقت أضرار بالغة بالمساهمين والدائنين والموردين والغير .

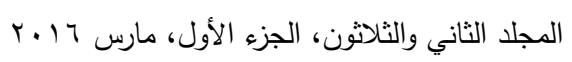




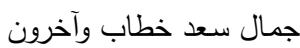

وبشكل عام يمكن القول بأن نظام الحوكمة يشكل دليلاً للرقابة الذاتية؛ أي كيف نتصرف

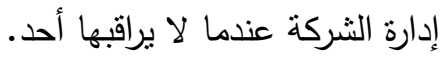

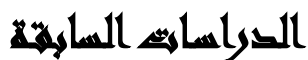

1 - دراسة (Black et al., 2003) بعنوان:: "تأثثير حوكمة الثركة على قيمة الثركة"، وقد شملت الدراسة الشركات الكورية الجنوبية المدرجة في السوق المالي، وقد تم استخدام أسلوب المسح الثامل للشركات المدرجة، وذلك بهدف إيجاد مؤشر للحاكمية المؤسسية،

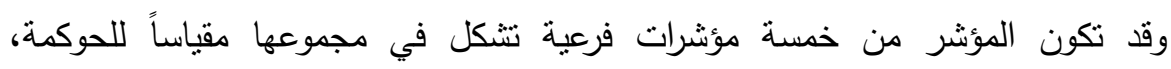
والمتمتلة في: (حقوق المساهمين، ومجلس الإدارة ولجنة التدقيق، والإفصاح، وتركز الملكية)، وتم اختيار أثز التباين في ممارسات الحوكمة في الثركات الكورية المدرجة في

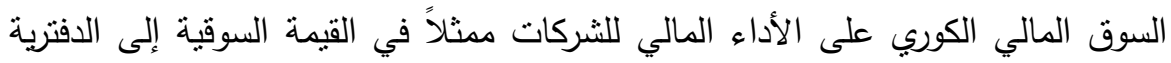
باستخدام تحليل الانحدار .

وقد توصلت الدراسة إلى أن هنالك علاقة بين مستوى الحوكمة في الثركة والقيمة

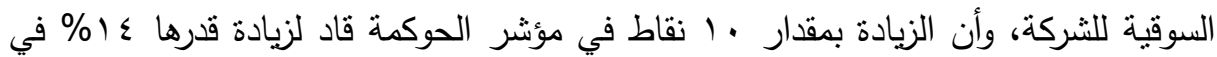
القيمة السوقية لسهم الثركة.

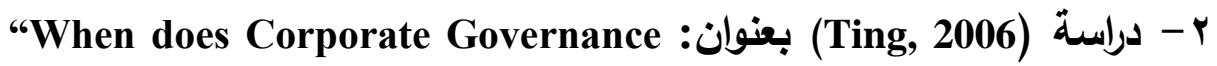
and Value"

تتاولت الدراسة موضوع: "متى تضيف حوكمة الشركة قيمة للشركة"، وقد أجريت

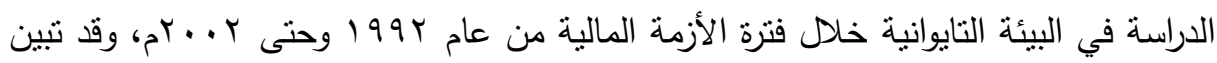
من خلال الدراسة الميدانية وجود أثز إيجابي لنظم الحوكمة على أداء الثئة الثركة الثرات.

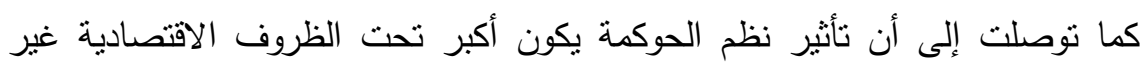
المواتية وتكاليف الوكالة المرتفعة والهيكل التتظيمي الأكثر تعقيداً، بالإضافة إلى أنى آنئ آليات نظام الحوكمة تعمل بكفاءة وفاعلية عندما يكون المدراء لديهم قناعة بأهمية الحوكمة. 
r - دراسة (عبل الرحمن، ب . . r) بعنوان: "الجوانب التحليلية والتطبيقية لمفهوم Corporate governance

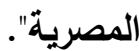

وقد تتاول الموضوع من خلال التعرض إلى مفهوم حوكمة الثركات ومدى إمكانية تطبيقه في الشركات المصرية، وخلصت إلى أن وسائل تقعيل الحوكمة تتلخص في تفعيل دور كل من المراجعة الخارجية، المراجعة الداخلية، الرقابة الداخلية، العمل على تطوير معايير المحاسبة والمراجعة، فصل مجلس الإدارة عن الإدارة التتفيذية، الاهتمام بالإفصاح والثفافية في القوائم المالية، تفعيل دور لجان المراجعة.

وتوصلت إلى أن تطبيق هذا المفهوم في البيئة المصرية من شأنه أن يؤدي إلى تفعيل عملية الإشراف والرقابة والمتابعة على الثركات المساهمة مما ينعكس على تحسين الأداء بهذه الثركات. كما يساعد نطبيق هذا النظام على زيادة الثفافية في القوائم المالية وحماية المساهمين وأصحاب المصالح الأخرى بالثركات. r- دراسة (ياسين، r +. ب) بعنوان: "دور الأجهزة الرقابية في تفعيل الإطار المحاسبي لحوكمة الشركات في مصر".

وذلك من خلال دراسة العلاقة بين الأجهزة الرقابية والمهنية وآلية التفاعل فيما بينها منل العلاقة التي تربط بين الهيئة العامة لسوق المال والهيئة العامة للاستثمار وبورصة الأوراق

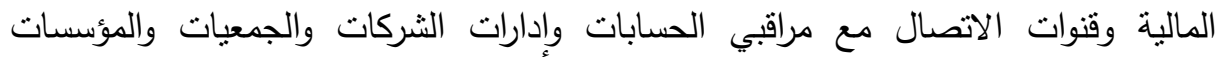
المحاسبية المهنية والجهاز المركزي للمحاسبات والبنك المركزي المصري بالإضافة إلى والى محاسبين قانونين غير مزاولين للمهنة منذ عثر سنوات للتأكد من مدى التزام مراقبي الحسابات

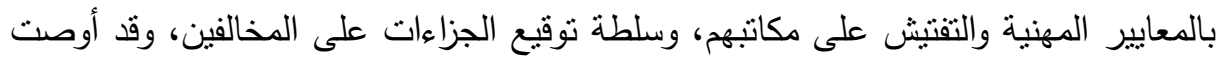

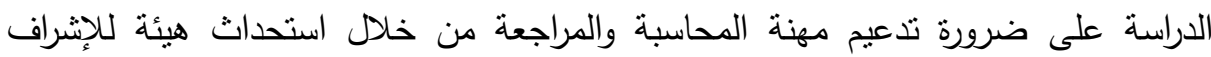

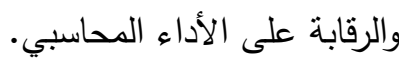




\section{مهرحلة الهميث}

على الرغم من أن النطور الفعلى لنظرية حوكمة الثركات لايزال يحوز اهتمام الكثير من

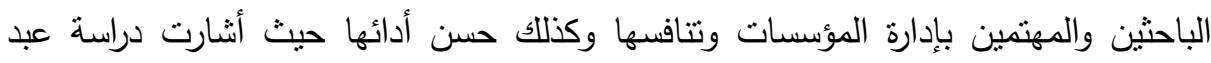
الرحمن r . . ب بعنوان الجوانب التحليلية والتطبيقية لمفهوم حوكمة الثركات وامكانية تطبيقها في الثركات العاملة في البيئة المصرية الي ان وسائل تفعيل الحوكمة تتلخص في تفعيل دور

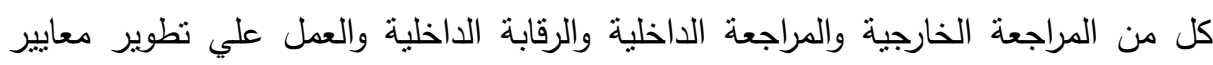

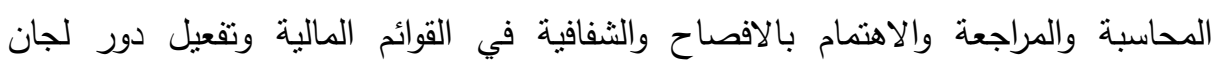
المراجعة وتساهم هذه الثفافية في حماية المساهمين وأصحاب المصالح الاخري بالثركات كما

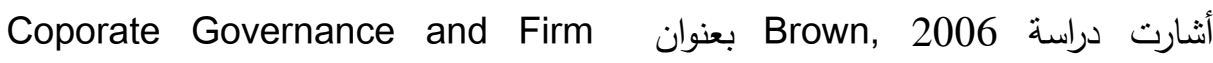
Valuation

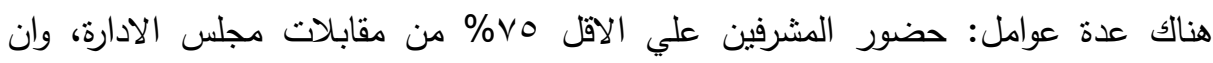

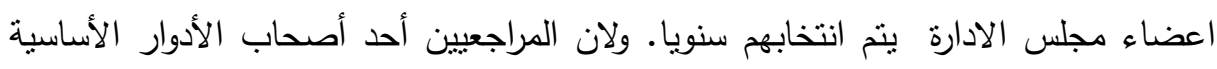
عند نطبيق أليات حوكمة الثركات فقد يتبادر إلى الباحثة السؤال التالى:

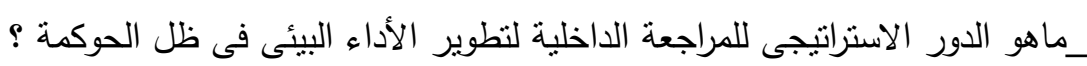
كما يتبادر إلى ذهن الباحثة مجموعة من الأسئلة الفرعية:

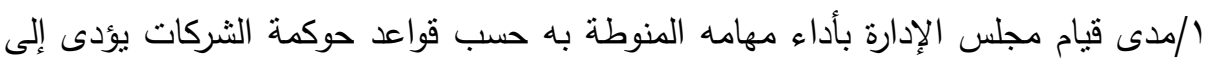
تحسين القرارات المالية الصادرة عند؟ الادرة باده مهاه

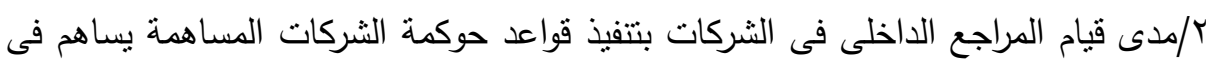
تحسين القرارات المالية الصادرة عن إدارات تلأك الثركات ؟

\section{أهمية الههمه}

تظهر أهمية البحث من خلال النقاط التالية: ا-تتبع أهمية البحث من الأهمية التي تحظى بها حكومة الثركات في الوقت الحاضر، وأهمية التدقيق الداخلي كونه أحد الأسس التي تركز عليها حوكمة الثركات من منطلق

$$
\text { الفصل بين الإدارة والملكية. }
$$

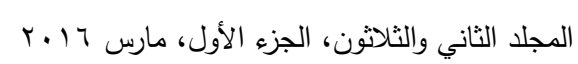


r-تساهم الدراسة في العمل على خلق حافز قوي للبدء بوضع مبادئ الحكومة في ليبيا.

\section{أهصاهض الههمه}

1- التعرف علي أثز تطور المعايير الدولية للممارسة المننية للمراجعة الداخلية فى دعم حوكمة الثركات . r- الوقوف على مدى التزام الثركات المساهمة العامة بتطبيق قواعد الحكومة.

\section{هروغر المهنه}

1- توجد علاقة ذات دلالة إحصائية بين قيام مجلس الإدارة بأداء مهامه المنوطة به حسب قواعد حوكمة الشركات المساهمة وبين تحسين القرارات المالية الصادرة عن إدارة الثركة. r- توجد علاقة ذات دلالة إحصائية بين قيام المراجع الداخلي في الثركات المساهمة العامة بتتفيذ قواعد حوكمة الثركات المساهمة، وبين تحسين القرارات المالية الصادرة عن إدارة الثركات.

r- توجد علاقة ذات دلالة إحصائية بين قيام المراجع الخارجي في الثركات المساهمة العامة بتتفيذ قواعد حوكمة الشركات المساهمة، وبين تحسين القرارات المالية الصادرة عن إدارة الثركات.

ع- توجد علاقة ذات دلالة إحصائية حول مدى التزام المراجع الداخلي والمراجع الخارجي بقواعد حوكمة الثركات المساهمة لتحسين القرارات المالية الصادرة عن مجلس إدل إدارة الثركة المساهمة في ليبيا.

\section{حبوض المهبه}

يتتاول هذا البحث الدور الإستراتيجى للمراجعة الداخلية لنطوير الأداء البيئى في ظل

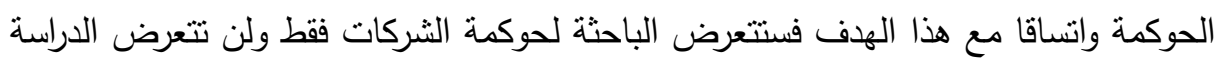

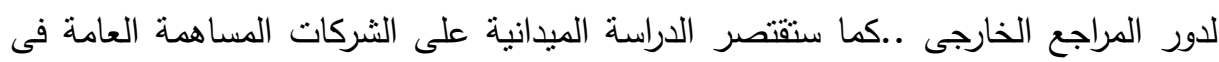

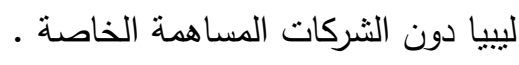




\section{هجنمع المهيش}

لقد اختير اسلوب الحصر الثامل وذلك لقلة عدد المراجعين الاخليين وكذلك قلة الثركات المساهمة العامة فى ليبيا وكان مجتمع وعينة الدراسة كالتالي: //بخصوص مراجعى الحسابات القانونيين الذى يتكون من مراجعى الحسابات القانونيين العاملين فى طرابلس وعددهم (70) مراجعا والمسجلين فى جدول المراجعين حسب دليل مراجعى الحسابات القانونيين الليبين الصادر عن جمعية المحاسبين والمراجعين الليبيين

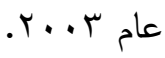

T/بخصوص مدراء الثركات المساهمة العامة حيث تثنمل على جميع الثركات المساهمة

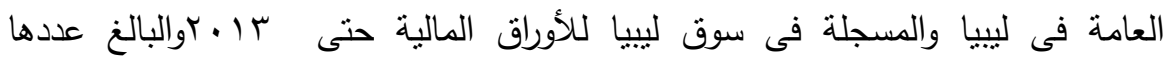

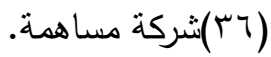

\section{الاطار المنظري الفوراسة}

أولاً: تظور تعريف وأهمية المراجعة الاخلية:

1 - تطور تعريف المراجعة الداخلية: ثانياً: أثر تطور مفهوم المراجعة الداخلية في دعم حوكمة المؤسسات: عند تتبعنا للعرض السابق حول تطور مفهوم المراجعة الداخلية وأهميتها نجد أن مجال

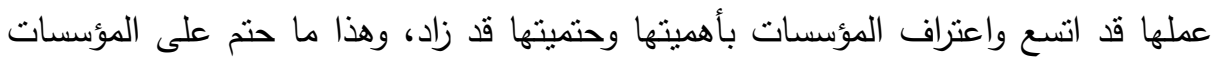
تعميم نظام للرقابة الداخلية فعال وكفاءة وهذا ما يؤدي إلى تطبيق حوكمة المؤسسات بشكل

ولبيان أثز تطور مفهوم المراجعة الداخلية في دعم حوكمة المؤسسات يرى كل من أن التعريف الجديد للمراجعة الداخلية يقدم صورة جديدة (Chapman and Anderson) للمهنة، في عدة اتجاهات رئيسية هي: 1- بأكيدية r ب المستقلة 


\section{r-استثارية \\ ع -موضوعية:}

ثالثاً: أثر تطور المعايير الدولية للممارسة المهنية للمراجعة الداخلية في دعم حوكمة المؤسسات:

من خلال المراجعة الداخلية السابقة للمعايير الدولية للممارسة المهنية الجديدة والقديمة

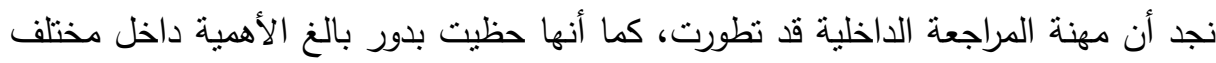

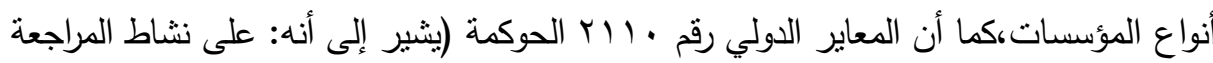
الداخلية المساهمة في تطوير عمليات الحوكمة بواسطة تقييم وتحسين هذه العطلية من خلال: 1-وضع القيم والأهداف والإعلان عنها. r- بمان فعالية إدارة المؤسسة والتأكد من المساءلة. r- إيصال المعلومات حول الرقابة والخط للجهات المعنية في المؤسسة.

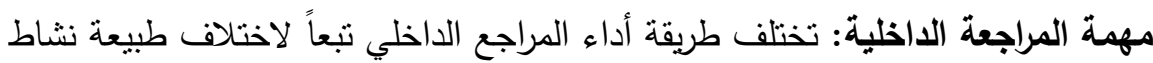

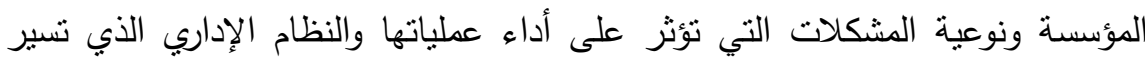
عليه إدارتها، إلا أن أداء مهمة المراجعة الداخلية يتم من خلال اختيار الجهة الخاضعة الهاه

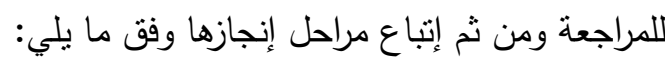
أولاً: اختيار الجهة الخاضعة للمراجعة: تبدأ مهمة المراجعة الداخلية باختبار النشاط الذي ولي سيخضع لعملية المراجعة والذي يتم وفق ثلاث طرق. 1-الاختيار المنظم: r-الاختيار بناء على طلب الإدارة العليا:

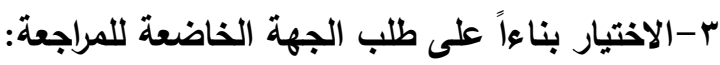
ثانياً: مراحل إنجاز عملية المراجعة الداخلية: 1-التخطيط لعملية المراجعة. r-الاتصال والموافقة. 
دور الأطراف الداخلية في حوكمة الثركات: يعتبر المراجع الداخلي أحد الأطراف التي تتمتع بدور متميز عند نطبيق آليات حوكمة الثركات على الرغم من أن مكانته الوظيفية وطبيعة الخدمات التي قدمها. قد تحد من قدرته على أداء الدور المطلوب منه. لذا ستتتاول الباحثة دور هذه الأطراف في تطبيق آليات حوكمة الثركات من خلال: - - دور المراجع الداخلي في حوكمة الثركات. - دور لجنة المراجعة في حوكمة الثركات.

دور المراجع الداخلي في حوكمة الثركات: إن وظيفة المراجعة الداخلية في بيئة الأعمال

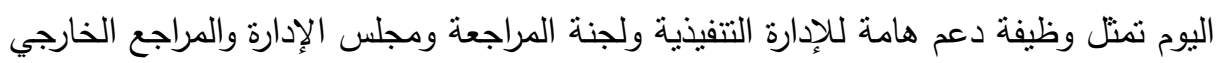

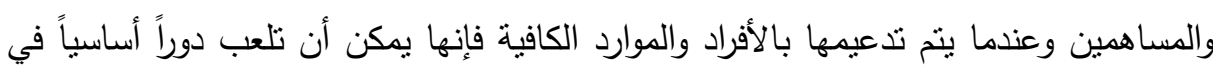
تطبيق حوكمة الثركات الفعالة.ونظراً لطبيعة أعمال البنوك وتعقدها وتداخلها تبرز أهمية وظيفة المراجعة الداخلية في المساعدة في تطبيق حوكمة الثركات من خلال قيام المراجع

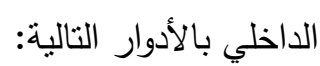
1- دور المراجع الداخلي في تقليم خدمات التأكيد عن نظم الرقابة:يعتبر دور المراجع

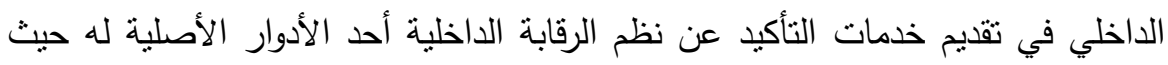

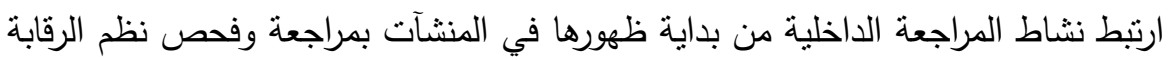

$$
\text { وتقديم تقرير بذلك إلى الإدارة التتفيذية. }
$$

r- تقييم نظم الرقابة الداخلية:يعتبر وجود الدليل الخاص بتوثيق نظم الرقابة هو المرجع الأساسي لعملية التقييم التي يقوم بها المراجع الداخلي حيث ينم التأكيد من مدى الالنزام

$$
\text { بتطبيق الاليل في الواقع. }
$$

\section{دور لجنة المراجعة في حوكمة الشركات:}

1 - أهمية لجنة المراجعة: نتيجة لحالات الفشل والتعثر التي تعرضت لها العديد من المنشآت والتي أظهرت القصور الواضح في أداء مجالس إدارة الثركات وانفراد بعض أعضه أعضاء مجالس الإدارة أو العضو المنتدب بجميع المسئوليات والصلاحيات في إدارة هذه الثركات

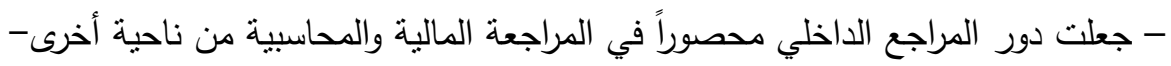

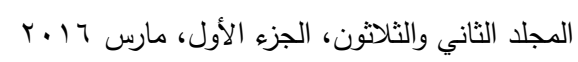


ويرى أن لجنة المراجعة تحقق الإثراف الفعال على عملية إعداد التقارير المالية وتكون أثز موضوعية عند تقييم

r - تثكيل لجنة المراجعة:وعادة ما تشكل هذه اللجان في الثركات المساهمة الكبيرة التي من طبيعتها التعامل مع عدد كبير من أصحاب حقوق الملكية ويوجد مدخلين منبعين في تثكيل لجنة المراجعة هما:

r- اختصاصات لجنة المراجعة:نظراً للأهمية التي تمنلها لجنة المراجعة في بيئة الأعمال

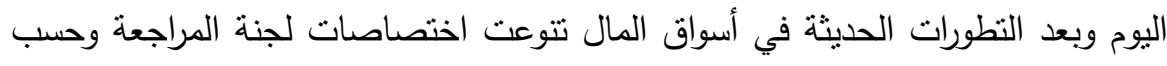
ما يرى البعض فإن هذه الاختصاصات يمكن تصنيفها كالآتي: - دمع عملية المراجعة الخارجية وتفعيل استقلاليتها. - الإشراف على إعداد التقارير المالية. - الإثراف على أعمال المراجعة الداخلية ونظم الرقابة الداخلية.

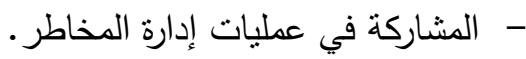
حوكمة الشركات (المفاهيم والخصائص): مفهوم حوكمة الثركات: مفهوم الحوكمة اصطلاحاً: لم تتلق الكتابات حول مفهوم واضح ومحدد. وقد رجح تتوع التعارف إلى تداخله في العديد من الأمور التتظيمية والاقتصادية والمالية والاجتماعية وهو الأمر الذي يؤثر على المجتمع والاقتصاد ككل. ويضيف أيضاً "هو الإجراءات المستخدمة بواسطة ممنلي أصحاب المئي المصلحة في

المنظمة لتوفير إثراف على المخاطر ورقابة المخاطر التي تقوم بها الإدارة".

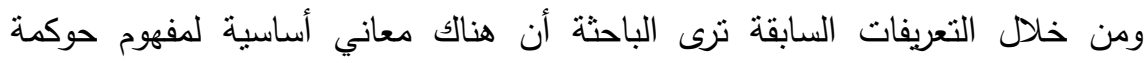

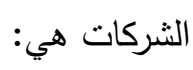
1-مجموعة من الأنظمة والقوانين والقواعد الخاصة بالرقابة على أداء الثركات. r-تتظيم للعلاقات بين مجلس الإدارة والمديرين والمساهمين وأصحاب المصالح الأخرى. 


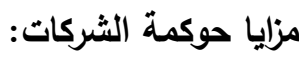

1-تخفيض المخاطر المتعلقة بالفساد المالي والإداري التي تواجهها الثركات والدول. r-رفع مستوى الأداء للشركات وما يترتب عليه من دفع عجلة التتمية والتقدم الاقتصادي للاول التي تتنمي إليها تلك الثركات. r-جذب الاستثمارات الأجنبية وتشجيع رأس المال المحلي على الاستثمار في المشروعات الوطنية وضمان تدفق الأموال المحلية والدولية. ع- حماية المساهمين بصفة عامة سواء أكانت أقلية أم أغلبية وتعظيم عائدهم. ه-ضمان مراجعة الأداء المالي وحسن استخدام أموال الثركة ومدى الالتزام بالقانون

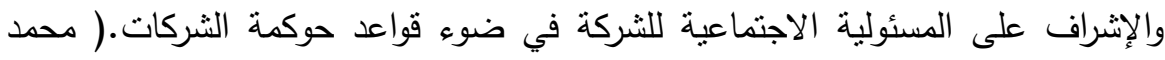

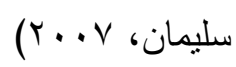

مبادئ حوكمة الثركات: سوف نتتاول عرض مبادئ منظمة التعاون الاقتصادي والتتمية

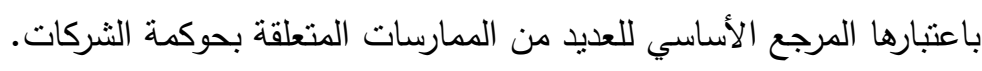
المبدأ الأول: ضمن وجود أساس لإطار فعال لحوكمة الثركات:ينص المبدأ الأول من مبادئ

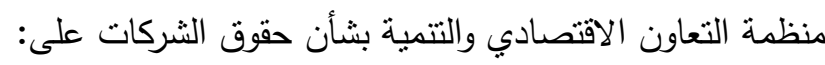
"ينبغي على إطار حوكمة الثركات أن يشجع على شفافية وفعالية وكفاءة الأسواق وأن أنسان يكون منوافقاً مع أحكام القانون وأن يحدد بوضوح توزيع المسئوليات بين مختلف الخدات الخبرات الاشتراطية والتتظيمية والتتفيذية".

المبدأ الثاني: حقوق المساهمين والوظائف الرئيسية لأصحاب حقوق الملكية: ينص المبدأ الثاني من مبادئ حوكمة الثركات: "ينبغي على إطار حوكمة الثركات أن يوفر الحماية للمساهمين وأن بسهل لهم ممارسة حقوقهم". أ- تشمل الحقوق الأساسية للمساهمين. ب- ينبغي أن يكون للمساهمين الحق في المشاركة وأن يتم إبلاغهم على نحو كاف بالقرارات المتعلقة بتغيرات أساسية في الثركة. 
الحوكمـة البيئية:ة

أولاً: مفهوم الحوكمة البيئية العالمية:

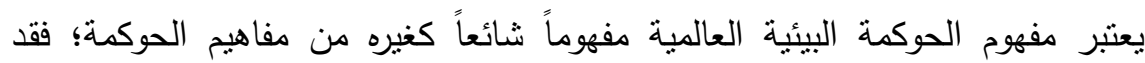
تعددت التعريفات المقدمة لهذا المفهوم لكنها تشترك في إبراز خصائص أساسية للحوكمة البيئية العالمية التي تؤكد على الأبعاد السياسية للضبط البيئي العالمي. (Paul, 2008)

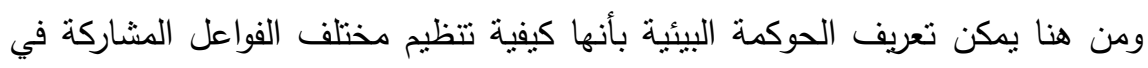
عمليات صنع القرار في المستوى العالمي، من أجل التحديات والنزاعات البيئية.

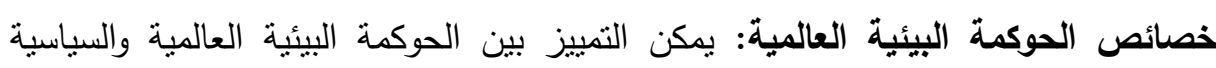
البيئية الدولية التقليدية من ثلاث خصائهة البئية العائص أساسية: أولاً: زيادة المشاركة: التنوع من خلال المشاركة: ثانياً: زيادة الخصخصة: التفاوض التفارئ التوع من خلال المشاركة: ثالثاً: زيادة التجزؤ: التعقد من خلادة التصفئ: التجزؤ: وظائف الحوكمة البيئية العالمية

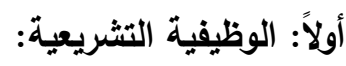

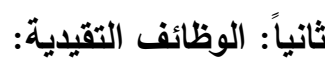
ثالثاً: الوظائف القضائية:

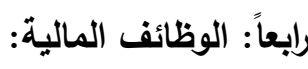

الدارسة التطبيقية

1 - منهجية الدراسة: من أجل نحقيق أهداف الدراسة نم استخدام المنهج الوصفي التحليلي والذي يعرف بأنه طريقة في البحث نتتاول أحداث وظواهر وممارسات موجودة متاحة للدراسة والقياس كما هي دون تدخل في مجرياتها، وتهدف هذه الدراسة إلى دراسة آراء المراجعين الخارجيين والمراجعين الداخليين مدراء الثركات في قياس مدى التزام الثركات المساهمة العامة في ليبيا بتطبيق قواعد حوكمة الثركات، وسوف نعتمد الدراسة على نوعين أساسيين من البيانات هما: 
أ- البيانات الثانوية: لقد قامت الباحثة بمراجعة الكتب والدوريات والمنشورات الخاصة أو المتعلقة بالموضوع قيد الدراسة، كذللك الكتب والمراجع العلمبة المتخصصة في المراجعة

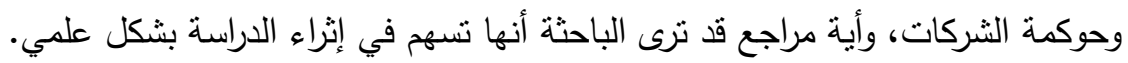

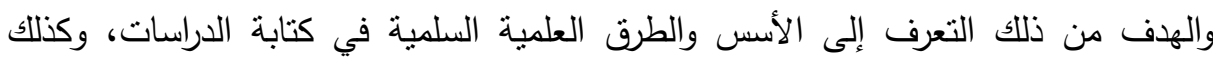
وضع تصور عام عن آخر المستجدات التي حدثت وتحدث في مجال الدراسة، ونظراً لعدم كفاية المصادر الثانوية تم اللجوء إلى البيانات الأولية.

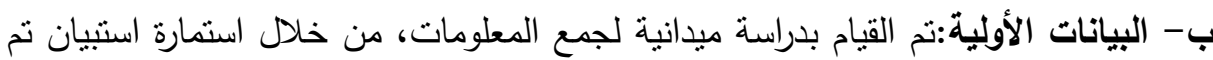
إعدادها لهذا الغرض، وتم تفريغ البيانات وتحليل النتائج باستخدام البرنامج الإحصائي .(SPSS- Statistical Package for Social Science) r - مجتمع وعينة الدراسة: لقد اختير أسلوب الحصر، وذلك لقلة عدد المراجعين الداخليين، وكذلك قلة الثركات المساهمة العامة في ليبيا، وكان مجتمع وعينة الدراسة كالتالي: أولاً: بخصوص مدققي الحسابات القانونيين الذي ينكون من مراجعي الحسابات القانونيين

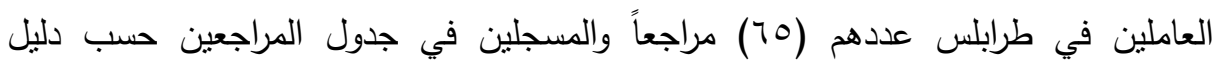
مراجعي الحسابات القانونيين اللبييين الصادرين عن جمعية المحاسبين والمراجعين الليبيين عام . . . r

ثانياً: بخصوص مدراء الثركات المساهمة العامة حيث تشنمل على جميع الثركات المساهمة

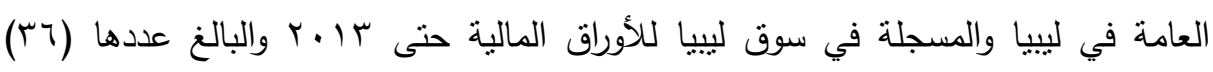
شركة مساهمة ثالثاً: بخصوص المراجعين الداخليين في الثركات المساهمة العامة حيث نتمل جميع

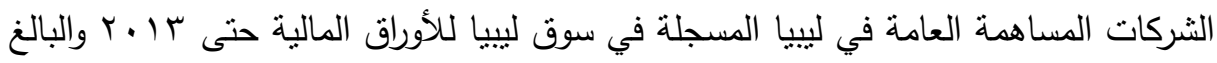
عددها (דب) شركة مساهمة. تم توزيع استمارات الاستبيان عليهم، وتم استرداد (1) استمارة، وبعد تقصص الاستمارات لم تنتبعد أي منها نظراً لتحقيق الشروط المطلوبة للإجابة عن الاستبانة، وبذلك لكان يكون عدد الاستبانات الخاضعة للدراسة (1) استمارة.

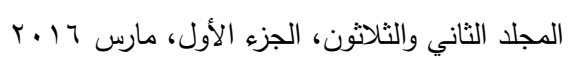


أولا: نتائج الدراسة الميدانية: اختبار التورزيع الطبيعي _اختبار كولمجروف - سمرنوف

(Sample K - S-1)

\begin{tabular}{|c|c|c|c|c|}
\hline المعنوية & $\begin{array}{c}\text { قيمة } \\
Z\end{array}$ & الفقرات & محتـوى المجــــال & المجال \\
\hline$\cdot, 091$ & $\cdot, V \vee Y$ & r $\varepsilon$ & 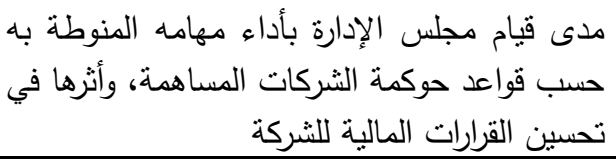 & الأول \\
\hline$\cdot, \wedge \wedge q$ & $\cdot, 0 \wedge 1$ & 10 & 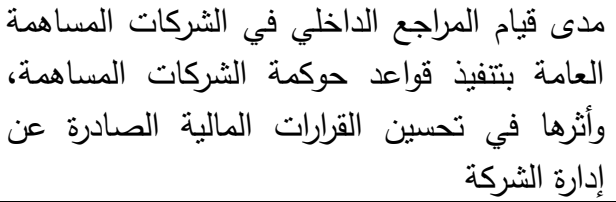 & الثاني \\
\hline$\cdot, \cdot \vee q$ & $1, r \vee 1$ & ir & 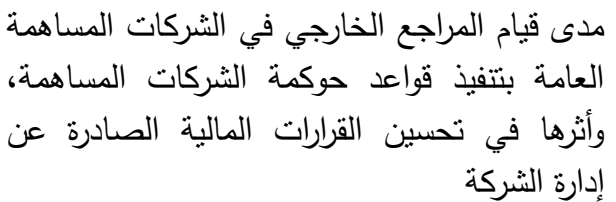 & 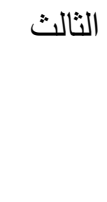 \\
\hline$\cdot, \wedge \vee \uparrow$ &., 091 & 01 & \multicolumn{2}{|c|}{ جمبـع الفقـرات } \\
\hline
\end{tabular}

ثانياً: تحليل ومناقثة نتائج اختبار فرضيات الدراسة:

في اختبار الفرضيات تم استخدام اختبار T للمجتمع الواحد One Sample T test) محتواها إذا كانت قيمة t المحسوبة أكبر من قيمة t الجدولية والتي تناوي 99, 1 (أو مستوى المعنوية أقل من ه., • والوزن النسبي أكبر من •ج\%ة)، وتكون الفقرة سلبية

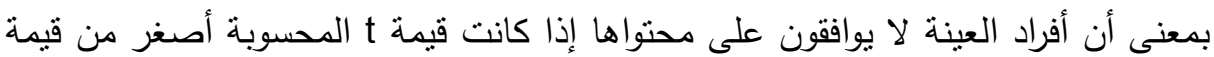

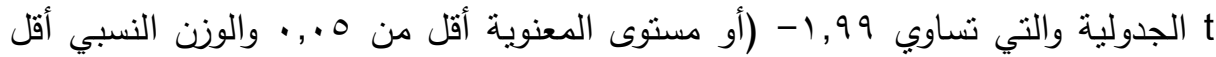
من · †\%)، وتكون آراء العينة في الفقرة محايدة إذا كان مستوى المعنوية أكبر من $\cdot, \cdot, 0$ 
جمال سعد خطاب وآخرون

يبين تحليل فقرات المجال الأول (مدى قيام مجلس الإدارة بأداء مهامه المنوطة به حسب قواعد حوكمة الثركات المساهمة، وأثرها في تحسين القرارات المالية للشركة)

\begin{tabular}{|c|c|c|c|c|c|}
\hline 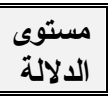 & t قيمة & 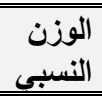 & المسابي & الفقــــــــرة & r \\
\hline$\cdot, \ldots$ & $r \cdot, 9 r$. & 91,10 & $\varepsilon, 09$ & يتم انتخاب مجلس إدارة الثركة من قبل الجمعية & 1 \\
\hline$\cdot, \cdots$ & $r\rceil, \leqslant Y \uparrow$ & $9 \cdot, \wedge \uparrow$ & $\varepsilon, 0 \leqslant$ & تقع المسؤولية النهائية في إدارة أمور الشركة على & $r$ \\
\hline$\cdot, \cdots$ & $r r, \varepsilon r \leq$ & $9 \cdot, 7 r$ & $\varepsilon$, or & 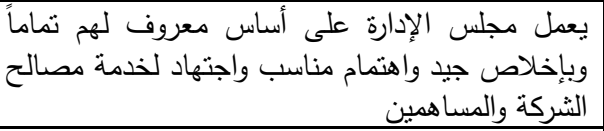 & r \\
\hline$\cdot, \cdots$ & IV,Tos & NV, 70 & $\varepsilon, \Gamma \wedge$ & 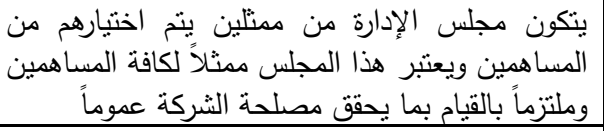 & $\varepsilon$ \\
\hline$\cdot, \cdots$ & $\wedge, \Gamma \wedge \wedge$ & $V V$, or & 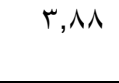 & المساهم مرشين عند دعوته الإدارة سيرة ذانتية مختصرة إلى المجلى & $\circ$ \\
\hline$\cdot, \cdots$ & $|7, r| r$ & $\Lambda \Lambda, Y_{0}$ & $\varepsilon, \varepsilon 1$ & وبلتزكل مجلس الإدارة بتطبيق القانون الأساسي للشركة & 7 \\
\hline$\cdot, \cdots$ & $9,9 \leftrightarrow \wedge$ & $\wedge 1,9 \wedge$ & $\varepsilon, 1$. & الإدارة الجديد بشفافية ونزارة الإدابق ترشيح وانتخاب مجلس & $\checkmark$ \\
\hline$\cdot, \cdots$ & 11,140 & $\wedge r, 0$. & $\varepsilon, 1 \wedge$ & يعامل مجلس الإدارة كل المساهمين بشكل عادل & $\wedge$ \\
\hline$\cdot, \cdot 1 \mathrm{~V}$ & $r, \Sigma \varepsilon$. & $\vee 7,1 \wedge$ & r T & الثشركات القانون الليبي لضرورة تطبيق قواعد حوكمة & 9 \\
\hline$\cdot, \cdots$ & $17, r \cdot 9$ & ᄉr,v. & $\varepsilon, 19$ & بطبق مجلس الإدارة معايير أخلاقية عالية في السلوك & 1. \\
\hline$\cdot, \cdots$ & $11, \ldots$ & $\Delta r, \ldots$ & $\varepsilon, 1$. & 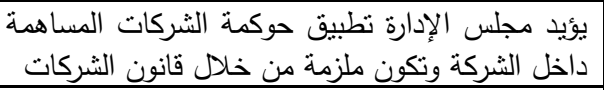 & 11 \\
\hline$\cdot, \cdots$ & Ir,vq. & $\wedge r, 97$ & $\varepsilon, 10$ & 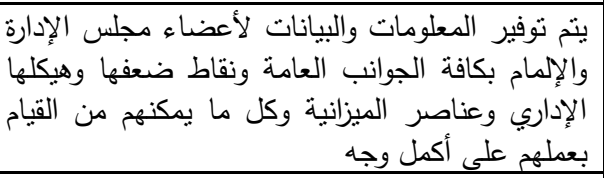 & IT \\
\hline$\cdot, \cdots$ & $11, r \cdot \varepsilon$ & $\wedge), \leqslant \Lambda$ & $\varepsilon, \cdot V$ & 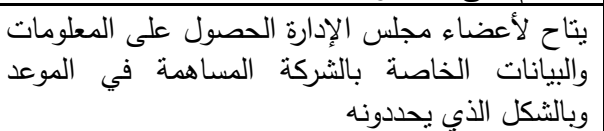 & $1 \pi$ \\
\hline$\cdot, \cdots$ & $\mid r, \ldots r$ & $\Lambda r, \leqslant \vee$ & $\varepsilon, 1 T$ & 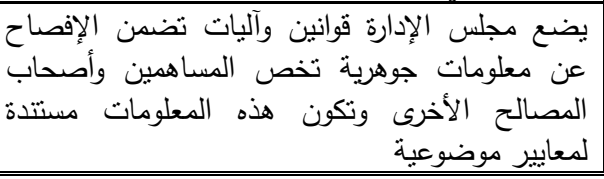 & $1 \varepsilon$ \\
\hline
\end{tabular}


مجلة العلوم البيئية

معهد الدراسات والبحوث البيئية - جامعة عين شمس لئس

\begin{tabular}{|c|c|c|c|c|c|}
\hline مستوى الدلالة & قيمة t & النسبي & الحستوبي & القة ــــــــرة & م \\
\hline$\cdot, \cdots$ & 11,709 & $\vee q, \leqslant q$ & r,qV & 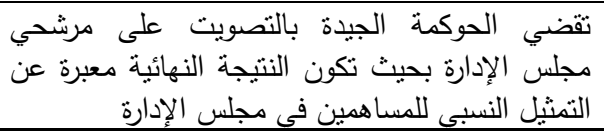 & 10 \\
\hline$\cdot, \cdots$ & $\wedge, \wedge \backslash 1$ & $\vee \wedge, O r$ & r,q & التتفكون غالبية أعضاء مجلس الثركة المساهمة الإدارة من الإدارة غير & 17 \\
\hline$\cdot, \cdots$ & $10,0 \mathrm{~s}$ & $\wedge r, \wedge$. & $\varepsilon, 19$ & 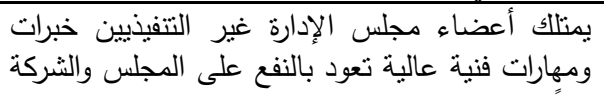 & IV \\
\hline$\cdot, \cdots$ & $10, \cdot 1 T$ & $10, \cdot 7$ & $\varepsilon, Y_{0}$ & 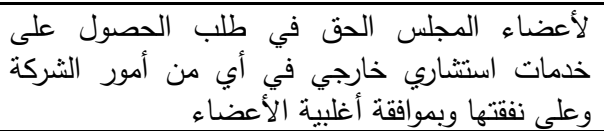 & 11 \\
\hline$\cdot, \cdots$ & $10,7 \mathrm{YV}$ & Ar,vo & $\leq, 1 \leq$ & 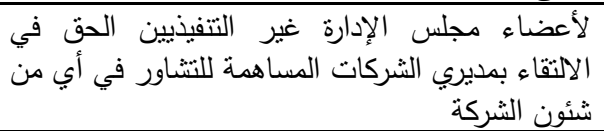 & 19 \\
\hline$\cdot, \cdots$ & $17,0 Y$. & $\wedge \varepsilon, \Gamma$. & $\varepsilon, Y, T$ & 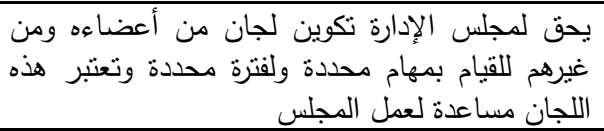 & $r$. \\
\hline$\cdot, \cdots$ & $r \cdot, \vee \wedge \uparrow$ & $\wedge \varepsilon, 0$. & $\varepsilon, Y T$ & 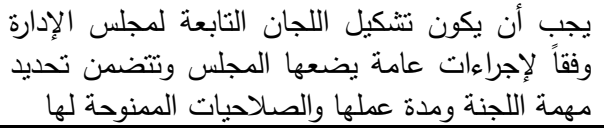 & YI \\
\hline$\cdot, \cdots$ & $\wedge, Y q \leq$ & $v\urcorner, \cdots$ & $\Gamma, \wedge$. & تشكل في الشركات المساهمة "لجنة للمراجعة" من عدد الإدارة غبر التففييين & Y \\
\hline$\cdot, \cdots$ & $18,11$. & $\Lambda T, Y_{0}$ & $\varepsilon, \Gamma$ & 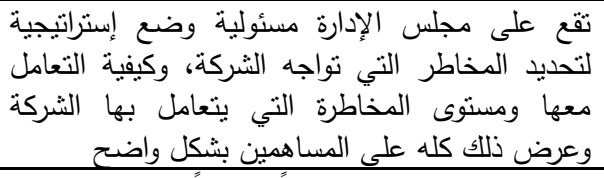 & rT \\
\hline$\cdot, \cdots$ & $r \varepsilon, V Y)$ & $91,0$. & $\varepsilon, 01$ & 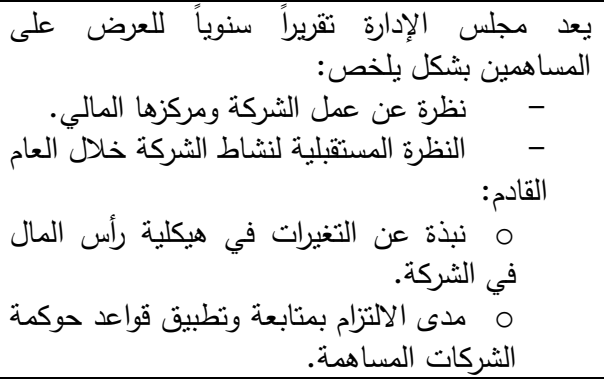 & $r \varepsilon$ \\
\hline$\cdot, \ldots$ & Yr,OYA & $\wedge r, o r$ & $\varepsilon, 1 \wedge$ & & جمي \\
\hline
\end{tabular}

قيمة t الجدولية عند درجة حرية (•^) ومستوى دلالة ه.ر. • تساوي 1,99 1 . 
r- تحليل فقرات المجال الثاني (مدى قيام المراجع الداخلي في الثركات المساهمة العامة

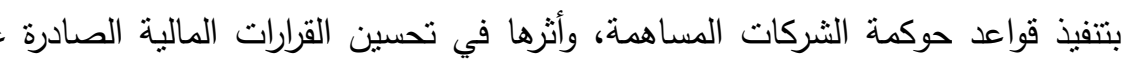

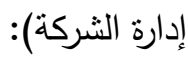

تم استخدام اختبار t للعينة الواحدة والنتائج مبينة في جدول رقم (10) والذي يبين أن

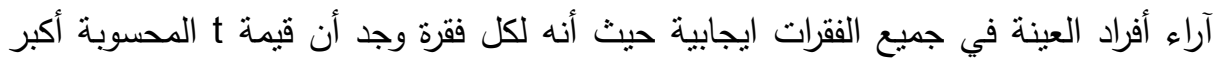

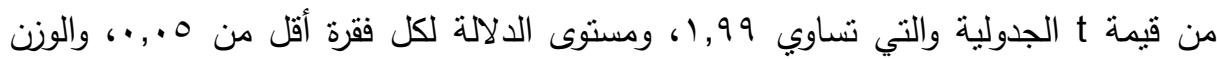

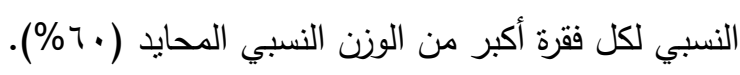

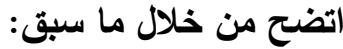
ا-تلتزم الثركات المساهمة العامة بالمبادئ المتعارف عليها لحوكمة الثركات مع أن التزامها بتلك المبادئ وجميعها وحدة واحدة هو بمستوى منوسط ويتفاوت مستوى الالتزام من مبدأ

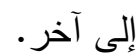
ץ-توجد علاقة إيجابية بين قيام كل من مجلس الإدارة والمراجع الداخلي والمراجع الخارجي

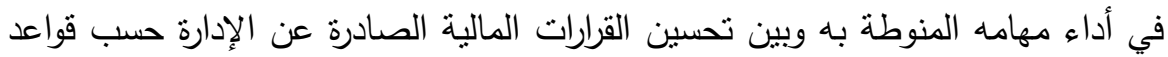
حوكمة الثركات بمساهمة العامة. r-يقدم مدير المراجع الداخلية تقريراً ربع سنوي إلى مجلس الإدارة عن مدى التزام الثركة المساهمة بحكام القانون والقواعد المنظمة لنشاطها. ع-يكون للثركة المساهمة مراجع حسابات خارجياً مستقلاً لا تربطه علاقة عمل ويكون مستقلاً عن إدارتها الداخلية بحيث يعمل المراجع ضد أي تدخل من قبل الإدارة في عمله.

\section{الميوسيايت}

1- العمل على إيجاد بيئة محاسبية ورقابية وتتظيمية سليمة تضمن وجود إدارات كفء ونزيهة

$$
\text { ومدققين يعملون باستقلالية ضمن المعايير المناسبة. }
$$

ץ- إلزام الثركات المساهمة العامة بتقييم مستوى حوكمة الثركات يجب ذلبن ذلك أن ينم من خلال تعديل قوانين الثركات بحيث تصبح إلزامية.

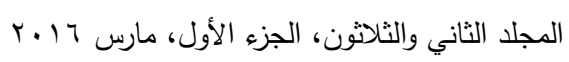


r-تشكيل لجان مستقلة داخل كل شركة تختص بوضع وتطبيق نظم التعيين والتقييم والترقية وتحديد الرواتب والمزايا والمكافآت.

\section{2ant}

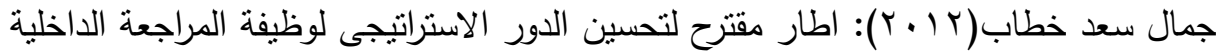

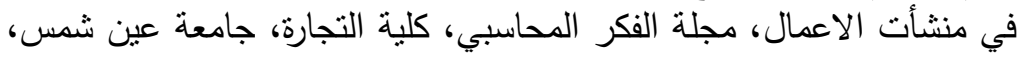

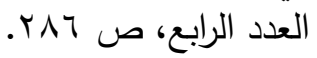

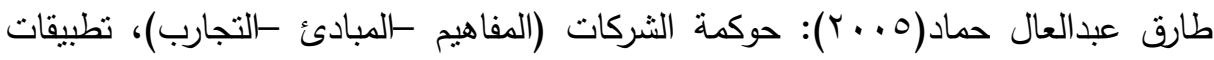

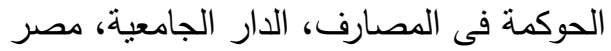

محمد عبد اللطيف خليل: دور المحاسب الاداري في إطار حوكمة الثركات ، مجلة الدراسات

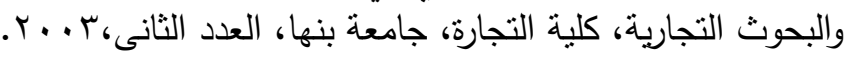

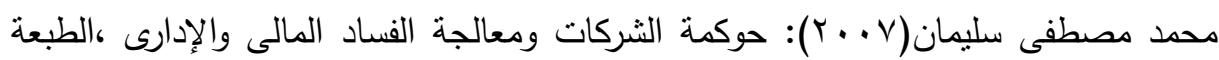
اللأولى، الدار الجامعية، الاسكندرية

يونس حسن عقل(0. . ب): تقييم دور الهيئة العامة لسوق المال فى تحسين فعالية التقرير

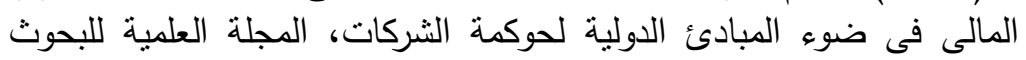
والدراسات التجارية، جامعة القاهرة، حلوان التانة

Paul A. Griffin, David H. Lont \& Yuan sun (2008): "Corporate Governance and Audit fees: Evidence of countervailing relations", Electronic copy available at: Http:/ssrn.com/abstract=1069480. 
جمال سعد خطاب وآخرون

\title{
THE STRATEGIC ROLE OF INTERNAL AUDITING \\ FOR DEVELOPING ENVIRONMENTAL \\ PERFORMANCE IN TERMS OF GOVERNANCE \\ AN ANALYTICAL STUDY ON LIBYAN \\ CORPORATIONS
}

Khatab, G. S. ${ }^{(1)}$; Matouq, M. A. ${ }^{(2)}$ Geberil, Magda, M. ${ }^{(1)}$

and Khalifa, Sanaa, A. ${ }^{(3)}$

1) Faculty of Commerce, Ain Shams University 2) Faculty of Economics, Tripoli University, Libya 2) Faculty of Economics and Political Science Bani Waleed University, Libya

\begin{abstract}
This study purpose is to expose the strategic role of internal auditing for developing environmental performance in terms of corporate' governance. A theoretical study and review of literaturesbased on form is designed consisting of four parts distributed on internal and external accounting auditors and public corporations managers. (137) questionnaire forms are distributed and (81) responses are obtained (60\%). The SPSS statistical program is used for analyzing data. The study comes to these results:

1- The public corporations are committed to known principles of corporate governance.

2- There are no auditing committees in Libya in corporations except for banks submitted to the control of environmental criticism authority.
\end{abstract}

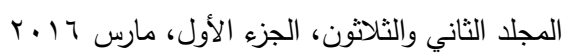

